\title{
Les crues de l'été 1987 dans les bassins versants glaciaires des Alpes Pennines
}

\author{
Yvon Rey et Georges Dayer *
}

\section{Introduction}

Les crues exceptionnelles de l'été 1987 ont fait l'objet d'une analyse dans le cadre des bassins glaciaires exploités par la société hydroélectrique Grande Dixence S.A. Ces bassins couvrent une surface de $360 \mathrm{~km}^{2}$ environ dans les Alpes Pennines, en Valais ; ils sont situés au-dessus de $2000 \mathrm{~m}$ d'altitude et se caractérisent par un taux de glaciation moyen de $60 \%$.

Pour situer l'ampleur de ces crues, il faut signaler qu'au niveau du Rhône à Sion (surface du bassin versant $3350 \mathrm{~km}^{2}$, taux de glaciation de $21 \%$ ), ces épisodes pluvieux extrêmes - combinés avec des volumes de fusion glaciaire déjà importants - correspondent à des crues d'un temps de retour de 200 ans environ pour la crue du mois d'août; ce temps de retour est estimé à partir de la loi de distribution de Gumbel, où l'on a reporté les débits annuels maxima observés depuis 1916. Par rapport à une situation analogue antérieure - soit en septembre 1948, oủ les digues du fleuve avaient cédé quelques kilomètres en aval de la ville - c'est 160 à $180 \mathrm{~m}^{3} / \mathrm{s}$ qui ont été soustraits aux débits du Rhône, par dérivations dans les réservoirs de montagne (ce qui correspond à $20 \%$ environ du débit maximum de la crue). Avant que ces crues d'origine pluviale ne se produisent, ces bassins glaciaires écoulaient déjà jusqu'à $800 \mathrm{l} / \mathrm{s} / \mathrm{km}^{2}$. Localement, $600-700 \mathrm{I} / \mathrm{s} / \mathrm{km}^{2}$ supplémentaires ont été apportés par les pluies; pendant plus de $24 \mathrm{~h}$. certains bassins ont ainsi écoulé en moyenne un total de $1400-1500 \mathrm{l} / \mathrm{s} / \mathrm{km}^{2}$.

\author{
La situation météorologique
}

Deux épisodes de crues distincts ont marqué l'été 1987. Un premier événement très localisé s'est produit les 1819 juillet ; le deuxième épisode du 24-25 août a touché une région plus étendue : ce dernier événement a causé sur l'ensemble de la Suisse - surtout en Suisse centrale - des dégâts estimés à près de 5 milliards de $\mathrm{FF}$.

Dans les deux cas, les situations météorologiques à l'origine de ces événements sont comparables. D'une part, une profonde dépression située sur l'Europe centrale et la Mer du Nord entraîne de l'air maritime plus frais vers les Alpes: le 23 août par exemple, les gradients de température à la surface de $850 \mathrm{hPa}$ sont remarquables : Edinburg $5{ }^{\circ} \mathrm{C}$, Francfort $18{ }^{\circ} \mathrm{C}$. Il faut toutefois noter que des irruptions d'air froid similaires - avec les perturbations pluvioorageuses qui leur sont associées - sont caractéristiques des mois de juillet-août. L'air froid pénétrant dans la région du golfe de Gênes arrive sur les arrières des masses d'air stationnaires plus chaudes et plus humides se trouvant sur la plaine du Pô. Les conditions optimales pour la genèse d'événements orageux extraordinaires sont alors réunies lors de la rencontre brutale de ces deux masses d'air : cyclonarité supplémentaire se concrétisant par une baisse de pression et un approfondissement de la dépression. Le 24 août, l'isotherme zéro degré était situé vers

(*) Grande Dixence S.A., rue des Creusets 41, 1951 Sion, Suisse.

The floods of 1987 in the glacial basins situated

in Pennine Alps in Switzerland

Exceptional rises of 1987 summer have been analysed in detail for some glacial basins situated in Pennine Alps in Switzerland. Precipitations of a recurrence time estimated between 80 and 100 years have affected localy some regions; these rains have increased the already large melting discharges resulting from hot days of July and August. Therefore, for more than 24 hours, some basins have drained off in average a total of 1,400 to $1,500 \mathrm{l} / \mathrm{s} / \mathrm{km}^{2}$, with some peaks estimated at 4,500-5,000 l/s/ $/ \mathrm{km}^{2}$. For the whole land of Valais, all the mountain reservoirs allowed to lower of about $20 \%$ the rise on the level with the river Rhone. 


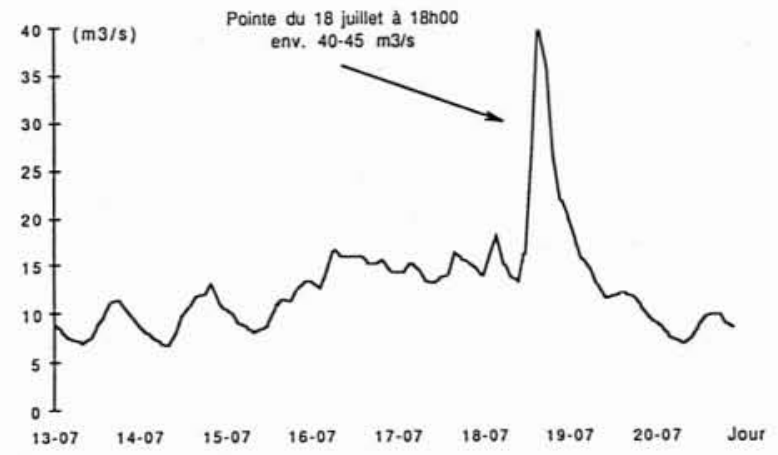

1. Débit horaire mesuré à l'Alpe de Stafel (du 13 juillet au 20 juillet 1987).

$3500 \mathrm{~m}$ : ainsi, en montagne, toutes les précipitations sont tombées sous forme de pluie et ont alimenté les crues en aval.

Pour mémoire, rappelons que la situation météorologique de la mi-juillet a été à l'origine de plusieurs catastrophes naturelles :

- crue du torrent de la Borne, débordements et désastre du Grand Bornand (14 juillet);

- en Italie septentrionale (Lombardie et Valteline), inondations catastrophiques du 17-19 juillet; ces mêmes précipitations sont responsables de l'effondrement ( rockslide ») d'une masse de 30 millions $\mathrm{m}^{3}$ de matériaux qui a barré la Haute Valteline et formé ainsi un barrage naturel pour les eaux de l'Adda.

\section{Les crues du 18-19 juillet 1987}

Tandis que des pluies extrêmement violentes s'abattaient sur la Suisse orientale principalement $(440 \mathrm{~mm}$ au Tessin en $48 \mathrm{~h}$ ), les bassins de montagne - où Grande Dixence S.A. exploite ses aménagements - étaient globalement épargnés : 10-20 mm ont été enregistrés dans les différentes vallées du sud des Alpes. Cependant, un épisode pluvieux d'une intensité exceptionnelle pour la région a eu lieu dans la zone de l'Alpe de Stafel et du glacier de Z'Mutt, sur un bassin versant de $35 \mathrm{~km}^{2}$ environ (taux de glaciation de $54 \%$ ). 55-60 mm de pluie ont été mesurés à Stafel en $24 \mathrm{~h}$, avec des intensités maximales dans l'après-midi du 18 juillet de $12 \mathrm{~mm} /$ heure. En plus de 25 ans d'exploitation, jamais Grande Dixence S.A. n'a été confrontée à de telles pluies, ni surtout à de telles intensités. Différentes estimations permettent d'évaluer le temps de retour d'une telle précipitation entre 80 et 100 ans; la valeur de cette fréquence découle aussi bien d'un ajustement des séries de mesures disponibles - soit 25 ans - à une loi de distribution de Gumbel que des cartes nationales des isohyètes des pluies centennales de $1 \mathrm{~h}$ et $24 \mathrm{~h}$. Ces pluies sont venues s'ajouter aux débits de fusion glaciaire importants provoqués par les chaudes journées de la première quinzaine de juillet : fonte du manteau neigeux superficiel, réorganisation et développement du réseau de drains sous-glaciaires sont deux éléments qui ont facilité les écoulements.

Pour ce bassin, les volumes écoulés en 24 h sont estimés à 1,8 million $\mathrm{m}^{3}$, qui se répartissent de la manière suivante :

- pour la partie glaciaire seulement, $900000-950000 \mathrm{~m}^{3}$ sont dus à l'ablation et à la fusion nivale, ce qui représente l'équivalent d'une lame d'eau écoulée de 100-120 mm. Cette valeur importante peut se comprendre si l'on y inclut les volumes des jours précédents stockés partiellement dans le manteau neigeux (50-60 $\mathrm{mm}$ de pluie en 4 jours $)$ - et relâchés avec la neige lors des fortes intensités du 18 juillet ; d'autre part, on peut estimer que la chaleur apportée par la pluie elle-même a contribué à la fusion glaciaire pour un total de $8-10 \mathrm{~mm}$.

- pour l'ensemble du bassin versant, $900000-950000 \mathrm{~m}^{3}$ sont les apports dus à la pluie uniquement, correspondant ainsi à une hauteur d'eau moyenne de $35-40 \mathrm{~mm}$.

En ce qui concerne la partie glaciaire du bassin versant uniquement, ces volumes correspondent en moyenne sur $24 \mathrm{~h}$ à un débit spécifique de $1600-1800 \mathrm{l} / \mathrm{s} / \mathrm{km}^{2}$; quant aux pointes de débit maximales, celles-ci peuvent être estimées à $4500-5000 \mathrm{l} / \mathrm{s} / \mathrm{km}^{2}$. Le temps de montée de l'hydrogramme de crue pour ce bassin est de $2-3 \mathrm{~h}$; le 18 juillet, la pointe de crue d'origine pluviale et le maximum de débit de fusion se sont produits simultanément à l'exutoire du bassin, provoquant ainsi un débit évalué à $40-45 \mathrm{~m}^{3} / \mathrm{s}$ (fig. 1). La moitié environ des volumes écoulés ont été soustraits par dérivation vers le lac des Dix; les volumes restants ont renforcé la crue du bassin aval.

\section{Les crues du 24-25 août 1987}

En cette saison, la ligne de névé est à sa cote la plus haute, se confondant ainsi avec la ligne d'équilibre du glacier; comme l'isotherme zéro degré moyen était situé vers $3500 \mathrm{~m}$ ce jour-là, c'est une surface glaciaire maximale qui a été touchée par cet épisode de pluie. Par analogie avec la situation du mois de juillet, ces apports se sont rajoutés aux débits de fusion importants des jours précédents. Enfin, les pluies du 23 août avaient déjà saturé - en partie du moins - les bassins non glaciaires situés en aval, favorisant dès lors un écoulement plus rapide pour les précipitations du 24 août.

Contrairement à l'épisode du mois de juillet, cette fois-ci les précipitations ont arrosé un territoire bien plus vaste, provoquant d'ailleurs ici et là des dégâts très importants. Le tableau 1 présente quelques caractéristiques des bassins glaciaires exploités par Grande Dixence S.A. : du glacier du Gorner au Val des Dix, on parcourt les Alpes Pennines de l'Est vers l'Ouest. Pour chacun de ces bassins, plusieurs éléments descriptifs des crues sont donnés : pluviométrie, volumes, débits spécifiques, etc... L'analyse de ce tableau permet de soulever les éléments de discussion suivants : 
CRUES DU 24 - 25 AOUT 1987

VOLUMES ECOULES ET DEBITS SPECIFIQUES

DES BASSINS VERSANTS GLACIAIRES EXPLOITES PAR GRANDE-DIXENCE

\begin{tabular}{|c|c|c|c|c|c|c|}
\hline & Gornera & $\begin{array}{l}\text { Alpe de } \\
\text { Stafel }\end{array}$ & $\begin{array}{l}\text { Vallon de } \\
\text { Ferpècle }\end{array}$ & $\begin{array}{c}\text { Haut } \\
\text { d'Arolla }\end{array}$ & 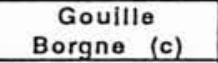 & $\begin{array}{l}\text { Val des } \\
\text { Dix }\end{array}$ \\
\hline $\begin{array}{l}\text { Surface } \\
\text { bassin versant (km2) } \\
\text { Glaciation } \\
\text { Altitudes } \\
\text { Orientation principale }\end{array}$ & $\begin{array}{c}80 \\
76 \% \\
1 ' 980-4.563 \mathrm{~m} \\
\text { Est - Ouest }\end{array}$ & $\begin{array}{c}34.5 \\
54 \% \\
22^{\prime} 213-4^{\prime} 476 \mathrm{~m} \\
\text { Ouest - Est }\end{array}$ & $\begin{array}{c}36 \\
64 \% \\
1 \cdot 910-4 \cdot 356 \mathrm{~m} \\
\text { Sud - Nord }\end{array}$ & $\begin{array}{c}12.9 \\
54 \% \\
2.483-3.838 \mathrm{~m} \\
\text { SE - NW }\end{array}$ & $\begin{array}{c}58 \\
37 \% \\
11^{\prime} 850-3^{\prime} 772 \mathrm{~m} \\
\text { Sud - Nord }\end{array}$ & $\begin{array}{c}44.5 \\
50 \% \\
2{ }^{\prime} 100-3.827 \mathrm{~m} \\
\text { Sud - Nord }\end{array}$ \\
\hline $\begin{array}{l}\text { Crues du } 24.08 .1987 \\
\text { Pluviométrie (mm/24h) } \\
\text { Volumes (mio m3/24h) } \\
\text { Hauteur équivalente } \\
\text { FUSION GLACIAIRE }(\mathrm{mm}) \\
\text { Hauteur équivalente } \\
\text { APPORTS PLUIES (mm) }\end{array}$ & $\begin{array}{l}\text { env. } 50 \\
2.9-3.2 \\
30-40 \\
25-30\end{array}$ & $\begin{array}{c}65 \\
1.78 \\
50 \cdot 60 \\
25 \cdot 30\end{array}$ & $\begin{array}{l}\text { env. } 45 \\
1.15 \\
50 \\
8 \cdot 10\end{array}$ & $\begin{array}{c}? ? \\
0.57 \\
54 \\
20-22\end{array}$ & $\begin{array}{c}50-60 \\
0.68 \cdot 0.76 \\
46 \\
20\end{array}$ & $\begin{array}{l}20-30 \\
0.95 \cdot 1.0 \\
20 \cdot 25 \\
8 \cdot 10\end{array}$ \\
\hline $\begin{array}{l}\text { Débits spécifiques } \\
\text { (1/s/km } 2) \\
\text { (moyennes sur } 24 \mathrm{~h})\end{array}$ & $650 \cdot 800$ & $850 \cdot 1^{\prime} 050$ & env. 680 & $850 \cdot 900$ & $780 \cdot 800$ & $350 \cdot 400$ \\
\hline $\begin{array}{l}\text { Crues maximales } \\
\text { antérieures (d) } \\
\text { Volumes (mio m3/24 h) }\end{array}$ & $3.1 \cdot 3.3(b)$ & $1.1-1.2,1.9 \quad$ (a) & $1.1 \cdot 1.4$ & $0.48-0.52$ & $0.33 \cdot 0.35$ & $0.9 \cdot 1.0$ \\
\hline
\end{tabular}

(a) Episode de foehn extrêmement violent (23.09.1981):

(b) Vidanges d'un lac glaciaire de confluence;

(c) Dérivations en amont de $4.2 \mathrm{~m} 3 / \mathrm{s}$ (0.36 mio m3/jour);

(d) En 20-25 ans d'exploitation des ouvrages de Grande Dixence.

Tableau No 1

- rôle du micro-climat: les contrastes d'origine topographique - et les micro-climats qui leur sont associés expliquent partiellement les différences d'ablation entre les appareils glaciaires: bassins intramontagneux largement influencés par le rayonnement terrestre, fonds de vallées encaissées, plongées dans l'ombre plus tard le matin et plus tôt l'après-midi, écarts considérables d'apports énergétiques entre adrets et ubacs, éventuelle formation d'inversions thermiques en altitude, etc... A ces raisons purement climatiques s'ajoutent les caractères des glaciers euxmêmes: orientation, pente moyenne, répartition de la surface en fonction de l'altitude, vitesse d'écoulement et renouvellement consécutif des surfaces soumises à l'ablation, développement différent du réseau de drains intra- et sous-glaciaires, glaciers "froids" et glaciers "chauds", etc... Les caractéristiques de l'écoulement à l'exutoire du bassin versant dépendent directement de l'ensemble de ces facteurs.

- illustration du rôle capital de la topographie. A quelques kilomètres de distance, deux vallées adjacentes reçoivent une quantité de pluie qui varie du simple au double: en effet, les masses d'air humides abandonnent de fortes quantités de pluie sur les flancs des massifs montagneux abordés sur leur trajectoire, et ces masses peuvent être d'une taille relativement restreinte — d'où le caractère local des orages.

- caractère extrêmement ponctuel des précipitations en montagne : («bulles» orageuses) : par exemple, les volumes réels apportés par les pluies dans le vallon de Ferpècle sont très inférieurs à ce à quoi on pouvait s'attendre à partir de la mesure de la hauteur de pluie. Il se pose dès lors la question de la représentativité d'une mesure de précipitations en montagne: le caractère fortement aléatoire dans le temps et l'espace des orages et l'effet du vent doivent toujours inciter à la prudence lors de l'interprétation de ces enregistrements !

- l'augmentation de la pluviosité avec l'altitude (gradient pluviométrique) ne se poursuit pas toujours vers le haut : en certains cas, les précipitations diminuent en fréquence et en abondance au-delà d'un niveau qu'on qualifie d'optimum pluviométrique. Dès lors, quel est l'influence de cet 
ELEMENTS D'ETUDE POUR LA CRUE DU 24 AOUT 1987 DANS LE VAL D'AROLLA

\begin{tabular}{|c|c|c|c|c|c|c|c|c|c|}
\hline & $\begin{array}{c}\text { Algullies } \\
\text { Rouges }\end{array}$ & $\begin{array}{l}\text { Fonta- } \\
\text { nesses }\end{array}$ & \begin{tabular}{|c|}
$\begin{array}{c}\text { Tsidjore } \\
\text { Nouve }\end{array}$ \\
\end{tabular} & Plèce & Vulbe & $\begin{array}{l}\begin{array}{l}\text { Bertol } \\
\text { inf. (a) }\end{array} \\
\end{array}$ & $\begin{array}{c}\text { Haut } \\
\text { Arolla } \\
\end{array}$ & \begin{tabular}{|c|} 
Bertol \\
supérleur
\end{tabular} & $\begin{array}{c}\text { Douves } \\
\text { Blanches }\end{array}$ \\
\hline Exposition & $E$ & $E \cdot N E$ & $N E$ & $N E$ & $\mathrm{~N}$ & $\mathrm{~N}$ & NW & SW & SW \\
\hline Surf. tot. (km2) & 7.4 & 4.8 & 4.8 & 2.7 & 2.1 & 8.2 & 12.9 & 2.5 & 1.4 \\
\hline Glaciation & $27 \%$ & $\cdots$ & $67 \%$ & $67 \%$ & $73 \%$ & $64 \%$ & $55 \%$ & $22 \%$ & $15 \%$ \\
\hline Vol. fusion & 85 & 14 (b) & 98 & 82 & 47 & env. 220 & env. 325 & 32 & 13 \\
\hline Lame fusion $(\mathrm{mm})$ & 45 & $\cdots$ & 34 & 45 & 35 & 42 & 54 & 56 & 59 \\
\hline $\begin{array}{l}\text { Vol. préc. } \\
\left(1^{\prime} 000 \mathrm{~m} 3\right)\end{array}$ & 70 & 94 (c) & 82 & 75 & 84 & $330-370$ & env. 245 & 61 & 34 \\
\hline Lame d'eau (mm) & 10 & $18-20$ & $18-20$ & $25 \cdot 30$ & env. 45 & $40-45$ & 22 & $24-26$ & $24 \cdot 26$ \\
\hline
\end{tabular}

- Volumes apportés par la fusion glaciaire et les précipitations du 24 août pour les différents sous-bassins exploitéspar Grande Dixence S.A. dans le Val d'Arolla.

(a) langues glaciaires jusqu'à 2'200 m environ.

(b) volume correspondant à un débit de base moyen de $160 \mathrm{l} / \mathrm{s}$.

(c) ce volume total se décompose ainsi : $611^{7} 700 \mathrm{~m} 3$ pour les premières 24 heures, $28^{\prime} 100 \mathrm{~m} 3$ pour le jour suivant et 4'600 m3 pour le 3-ième jour.

Tableau No 2

optimum dans le cadre d'un épisode de pluie ponctuel? II faut d'ailleurs souligner que la question de l'existence même de cet optimum dans les Alpes est controversée : pour certains, cet optimum se placerait d'ordinaire entre 300 et $500 \mathrm{~m}$ en dessous des plus hauts sommets, en raison d'une ascendance moins active vers les cimes. D'autres auteurs nient l'existence de ce maximum pluviométrique, en arguant du fait que les observations sur les sommets battus par les vents n'étaient pas correctes.

De manière générale, les volumes écoulés lors des crues du 24 août sont supérieurs ou égaux aux volumes mesurés depuis 1960-1965, même si l'on considère les phénomènes naturels exceptionnels qui ont provoqué ces crues dans le passé : vidanges d'un lac de confluence glaciaire, "sacs» d'eau dus à des orages de foehn, etc... Cependant, les débits écoulés dans la Borgne - au lieu-dit La Gouille - correspondent à plus du double des maximas enregistrés. Pour le bassin situé en amont de cette station, le tableau 2 présente quelques éléments d'analyse pour les différents sous-bassins exploités par Grande Dixence S.A. Dans ce cas, on ne peut pas déterminer si les versants orientés vers l'Ouest ont été plus ou moins arrosés que les versants exposés à l'Est ; par contre, la fusion de la glace est proportionnellement plus importante pour les bassins d'exposition Ouest ou Sud-Ouest.

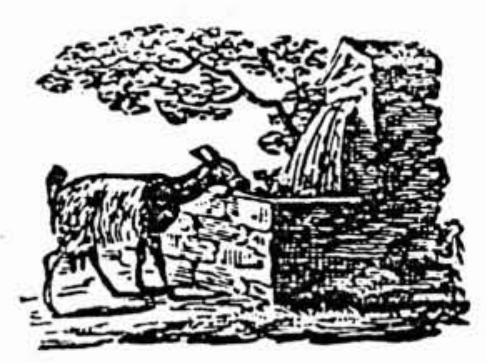




\section{Conclusion}

En ce qui concerne les aménagements de Grande Dixence seulement, les dommages les plus importants occasionnés par ces crues sont les suivants :

- passes des digues d'un bassin de décantation emportées, bassin de compensation d'une usine de pompage comblé de matériel morainique; d'autres bassins de réglage ont été partiellement remplis de matériaux, occasionnant ultérieurement des usures plus importantes sur les marchines ;

- prises d'eau obstruées dans la zone des adductions et dégradations importantes des stations de mesures;

- ponts touristiques emportés dans le Val d'Arolla et route d'accès emportée sur une distance de $300 \mathrm{~m}$;

- déversements dans les virages d'une rivière endiguée localement, à travers le village de Zermatt.
Les ouvrages de Grande Dixence S.A. ont permis d'écrêter les crues de plusieurs rivières de montagne (Vispa et Borgne) ; sans ses ouvrages de dérivation et de retenue, les dommages occasionnés en aval par ces crues auraient été bien plus importants : le Rhône qui collecte toutes les eaux des vallées alpines a également bénéficié - au niveau du plan d'eau, vitesses d'écoulement, érosion des berges, etc... - de cette réduction de débit.

Si des crues semblables se produisaient lorsque le niveau du lac est proche du maximum, différentes mesures préventives "en cascade" sont prévues, et leur mise en œuvre permettrait de maîtriser la montée du plan d'eau de la retenue du lac des Dix - au détriment toutefois d'un renforcement des crues dans les rivières de montagne et dans le Rhône.

Sion, le 3 novembre 1989

\section{Références}

ASCHWANDEN H., SCHÄDLER B., 1988: "Hochwasserereignisse im Jahre 1987 ", LHG-Mitteilung Nr. 10, Bundesamt für Umwelschutz, Bern.

BOuĖt M., 1972 : "Climat et météorologie de la Suisse romande », Payot Lausanne.

Estienne P., Godard A., 1970 : “ Climatologie », Armand Colin, Paris.

REY Y., DAYER G. : " Discharge forecasting from glacierized areas to optimize hydroelectric power management ", 25th International Geographical Congress in Applied Geography, Zürich, 1984.

Schweizerische Meteorologische Anstalt: "Ergebnisse des täglichen Niederschlagsmessung auf den meteorologischen und Regenmess-Stationen der Schweiz " ; erscheint quartalsweise, Zürich.

Zeller J., Geiger H., Röthilisberger G., 1976: “ Précipitations extrêmes dans les Alpes suisses et leurs régions limitrophes". Bd. 5, Eidg. Anstalt für das forstliche Versuchswesen, Birmensdorf. 


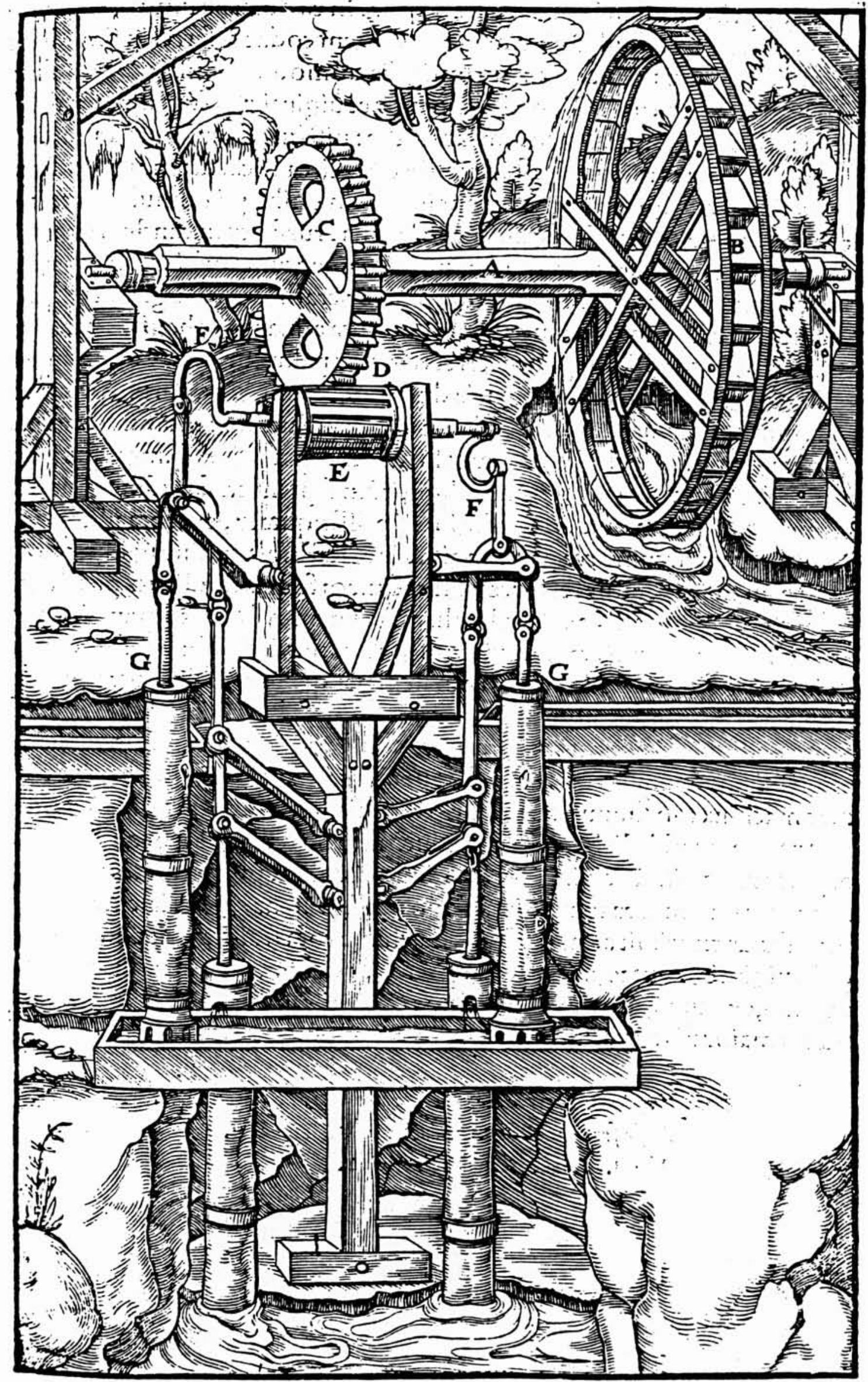

Extrait de: Agricola - De re metallica (1556)

LA HOUILLE BLANCHE/N $5-1990$ 